\title{
Intervertebral Disc Extrusion between T8 and T9 in a Dachshund
}

\author{
Gabriel Antonio Covino Diamante', Paulo Vinicius Tertuliano Marinho², \\ Carolina Camargo Zani' ${ }^{1}$, Bruno Cesar Elias ${ }^{1}$ \& Mônica Vicky Bahr Arias ${ }^{3}$
}

\begin{abstract}
Background: Intervertebral disc extrusion with consequent spinal compression or intervertebral disc disease (IVDD) is one of the most common causes of compressive myelopathy in dogs, and the thoracolumbar spine discs between T12 and L2 are most affected. Extrusions in cranial thoracic region are rare, and there is few cases in literature reporting this situations, this rarity is attributed to the presence of the intercapital ligament connecting the rib heads between T2 and T10, which strengthens this region both mechanically and anatomically. The aim of this article is report the clinical signs, diagnosis and treatment of a case of Type I Hansen IVDD between T8 and T9 in a Dachshund breed dog.

Case: An 8-year-old Dachshund male dog was presented for investigation of pelvic limb incoordination and back pain, which started 10 days prior to the consultation. It had a history of a similar condition 3 months earlier that showed improvement after clinical treatment. On neurological examination was identified in both pelvic limbs proprioceptive ataxia, absence of proprioception, increased muscle tone, presence of interdigital reflex and increased patellar reflex. Cutaneous trunci reflex was absent below T11 on the left side, and pain was noted upon palpation of T7 through the T12 vertebrae. A grade II asymmetric thoracolumbar lesion with hyperesthesia was diagnosed. On suspicion of IVDD, cerebrospinal fluid (CSF) collection and CT scan analysis of the thoracolumbar region were performed. CT scan showed the presence of hyperattenuating and mineralized material, $1 \mathrm{~cm}$ long, on the floor of the spinal canal, mainly on the left side, occupying $80 \%$ of the diameter of the spinal canal between T8 and T9. The patient was then submitted to decompression surgery through hemilaminectomy and showed a good recovery. As the occurrence of disc extrusion in cranial thoracic region of chondrodystrophic breeds is rare, we report the clinical signs, diagnosis and treatment of a case of Type I Hansen intervertebral disc disease (IVDD) between T8 and T9 in a Dachshund breed dog.

Discussion: All spinal discs can undergo a process of degeneration causing IVDD, in which the cervical and thoracolumbar regions are the most affected due to reasons not yet fully understood, however spinal cord compression between the $\mathrm{T} 1$ and $\mathrm{T} 9$ vertebrae is very rare, probably due to the strengthening provided by the intercapital ligament to the posterior annulus fibrosus. Disc extrusion in unusual locations in chondrodystrophic breeds is mainly reported as extrusion between vertebrae $\mathrm{T} 1$ and $\mathrm{T} 2$, and $\mathrm{T} 9$ and $\mathrm{T} 10$, with some cases was attributed to an anatomical abnormality of the intercapital ligament. The evaluation of intervertebral discs of the German shepherd breed dogs using MRI showed disc degeneration processes in thoracic vertebras. Disc extrusions often result in more severe clinical signs than protrusions, and occur acutely or subacutely, which was different from the case described here, maybe because the presence of the intercapital ligament permitted gradual extrusion allowing the spinal cord to adapt to the compression. Despite surgical access to this region being described as more complex due to the presence and proximity of the rib head to the vertebral body and the possibility of injuring the intercostal muscles causing pneumothorax, the hemilaminectomy and rib head excision at T9 could be performed without any complications. Thus, although unusual, the cranial thoracic region should not be overlooked as a possible site of occurrence of IVDD, since the diagnosis, treatment and prognosis are similar to those in patients with extrusions in the most common sites.
\end{abstract}

Keywords: intervertebral disc degeneration, dogs, paresis, ataxia. 


\section{INTRODUCTION}

Intervertebral disc extrusion with consequent spinal compression, or intervertebral disc disease (IVDD), is one of the most common causes of compressive myelopathy in dogs, and the thoracolumbar spine discs between T12 and L2 are most affected [9]. Extrusions in the cranial thoracic region are very rare, which is attributed to the presence of the intercapital ligament connecting the rib heads between $\mathrm{T} 2$ and T10. Anatomically, the ligament lie immediately dorsal to the annulus fibrosus and ventrally to the dorsal longitudinal ligament, which strengthens this region both mechanically and anatomically [2-3,12]. In nonchondrodystrophic breeds, especially German Shepherds, herniations are reported at T2-T3, T3-T4 and T4-T5 [3,7], while chondrodystrophic breeds are reported to have sporadic extrusions at T1-T2 and T9-T10 [8,12].

The occurrence of disc extrusion in the thoracic region of chondrodystrophic breeds is rarely reported. The aim of this article is to report the clinical signs, diagnosis and treatment of a case of Type I Hansen IVDD between T8 and T9 in a Dachshund breed dog.

\section{CASE}

An 8-year-old castrated male Dachshund dog was presented for investigation of apathy, decreased physical activity, pelvic limb incoordination and back pain that had progressed over the 10-days preceding consultation. The patient presented similar clinical signs 3 months prior to the current consultation and showed improvement after treatment with rest, acupuncture, non-steroidal anti-inflammatory drugs, and painkillers.

A neurological exam showed kyphosis, proprioceptive ataxia, and absence of proprioception in the pelvic limbs. Cranial nerves and thoracic limbs were normal; however, the pelvic limbs showed increased muscle tone, presence of interdigital reflex and increased patellar reflex. Cutaneous trunci reflex was absent below T11 on the left side, and pain was noted upon palpation of $\mathrm{T} 7$ through the $\mathrm{T} 12$ vertebrae. The signs were compatible with Grade II asymmetric thoracolumbar disc disease with thoracolumbar hyperesthesia.

On suspicion of IVDD, cerebrospinal fluid (CSF) collection and CT scan analysis of the tho- racolumbar region were performed. CSF analysis showed a mild increase in protein levels without pleocytosis. Sagittal, transverse and frontal images CT scan showed the presence of hyperattenuating and mineralized material, $1 \mathrm{~cm}$ long, on the floor of the spinal canal, mainly on the left side, occupying $80 \%$ of the diameter of the spinal canal between $\mathrm{T} 8$ and T9 (Figure 1). It also showed the presence of a small amount of hyperattenuating and mineralized material in the ventral portion of the spinal canal, between L2-L3, occupying $10 \%$ of it.

The patient was then submitted to decompression surgery through hemilaminectomy after being premedicated with $0.05 \mathrm{mg} / \mathrm{kg}$ acepromazine ${ }^{1}$ and $0.5 \mathrm{mg} /$ $\mathrm{kg}$ morphine ${ }^{2}$. Anaesthesia was induced with $0.3 \mathrm{mg} /$ $\mathrm{kg}$ midazolam ${ }^{3}$ and $3 \mathrm{mg} / \mathrm{kg}$ propofol ${ }^{4}$ and maintained with isoflurane ${ }^{5}$ and oxygen. Antibiotic prophylaxis was performed with $22 \mathrm{mg} / \mathrm{kg}$ cefazolin ${ }^{6} 30$ min prior to the start of the procedure and again every $8 \mathrm{~h}$ after the first dose for $24 \mathrm{~h}$.

Left dorsolateral hemilaminectomy between the T8 and T9 vertebrae, as described in the literature for the caudal thoracic region [11], associated with the removal of the $\mathrm{T} 9$ rib head, was conducted with a high-speed electric drill with round burrs, and rongeurs. The epaxial muscle (Longissimus dorsi and iliocostalis) were cautiously dissected to avoid laceration that could penetrate the chest cavity and lead to iatrogenic pneumothorax. A sandy and calcified material, which was compatible with nucleus pulposus extrusion, was found between $\mathrm{T} 8$ and T9. The disc material was removed until the spinal cord was completely decompressed (Figure 2 ), and the surgical site was closed. Postoperative management consisted of $4 \mathrm{mg} / \mathrm{kg}$ Tramadol ${ }^{7}$ every $8 \mathrm{~h}$ for 5 days and $0.5 \mathrm{mg} / \mathrm{kg}$ Meloxicam ${ }^{8}$ every 24 h for 3 days.

The patient was evaluated 24 and $72 \mathrm{~h}$ after the surgery and showed improvement in pain; however, the proprioceptive ataxia remained. Examination ten days after the surgical procedure revealed clinical improvement and only mild proprioceptive ataxia remained. Forty days following the surgery the patient returned to normal activities without pain and had only mild left pelvic limb incoordination. After 80 days, the patient presented full motion recovery. 


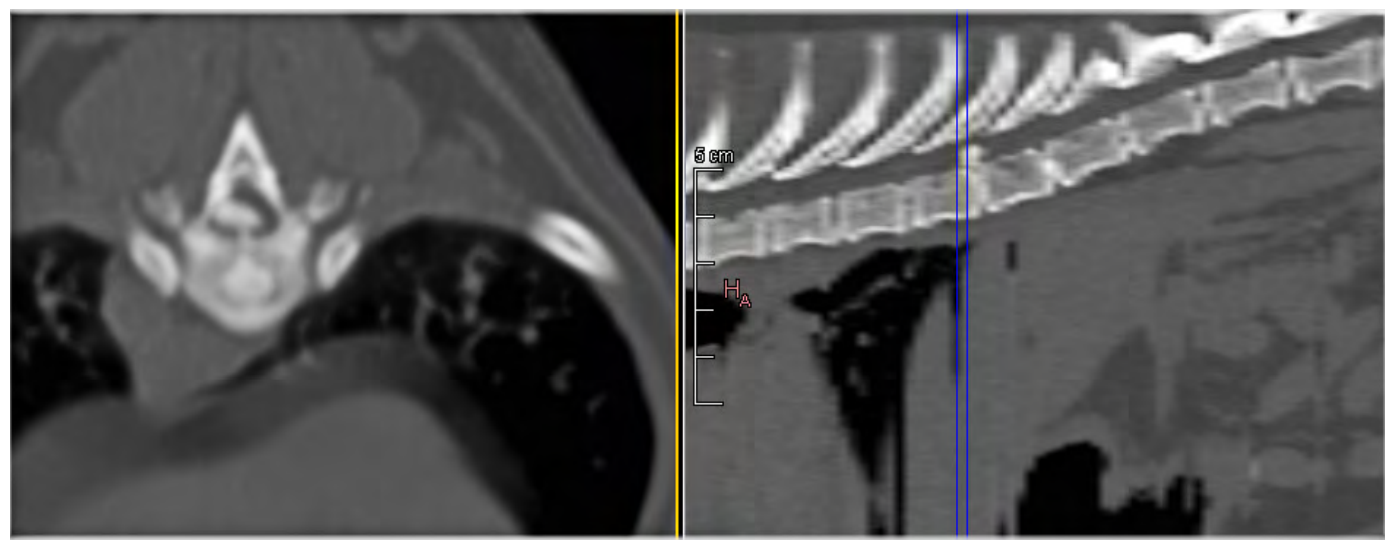

Figure 1. Sagittal (A) and transverse (B) images of CT scan showing presence of hyperattenuating and mineralized material, on the floor of the spinal canal between T8 and T9, mainly on the left side, occupying $80 \%$ of the diameter of the spinal canal.

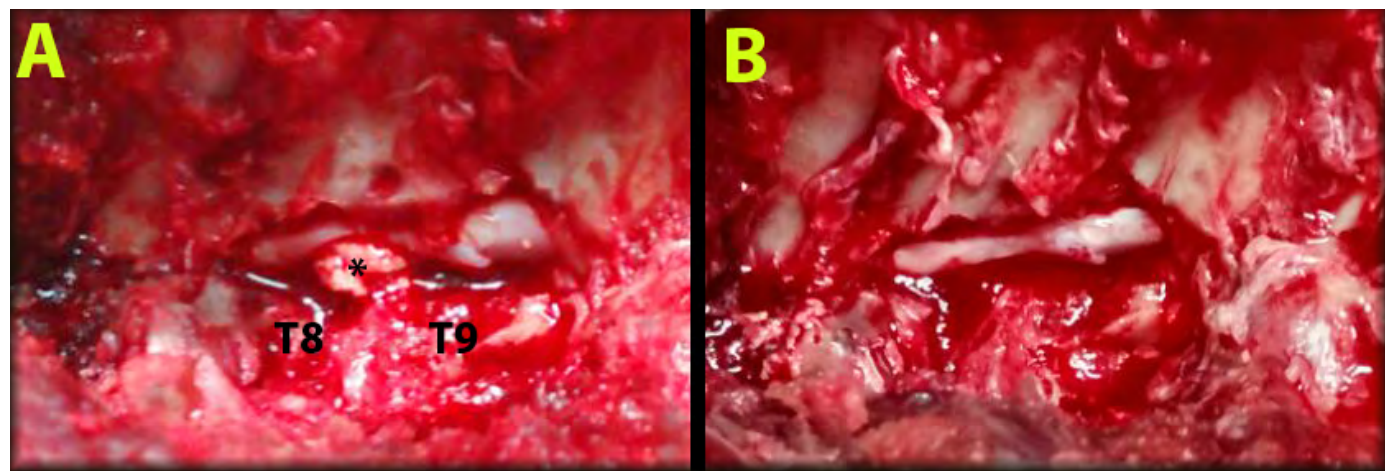

Figure 2. *A sandy and calcified material compatible with nucleus pulposus extrusion, compressing the spinal cord, between T8 and T9 (A). Spinal cord completely decompressed after the disc material has been removed (B).

\section{DISCUSSION}

All spinal discs can undergo a process of degeneration; however, the occurrence of extrusions is more common in chondrodystrophic patients, in which the cervical and thoracolumbar regions are the most affected due to reasons not yet fully understood [1].

Although disc degeneration can occur in any region of the spine, spinal cord compression between the T1 and T9 vertebrae is very rare [6], probably due to the strengthening provided by the intercapital ligament to the posterior annulus fibrosus [6,8]. Disc extrusion in unusual locations in chondrodystrophic breeds is mainly reported as extrusion between vertebrae $\mathrm{T} 1$ and $\mathrm{T} 2$ [5,8] and T9 and T10 [12]. We did not find any reports of extrusion at vertebrae T8-T9. In three Dachshunds with extrusion at T9-T10, the occurrence was attributed to an anatomical abnormality of the intercapital ligament, but similar to that in our patient, assessing the ligament during surgery was not possible [12].
Cranial thoracic compressions are more common in large breeds: in 723 dogs with IVDD, none of the 527 small breed dogs presented IVDD in the cranial thoracic region, whereas out of the 196 large breed dogs, 21 presented with the condition, mainly at T2-T3 and T4-T5, with $52.4 \%$ of the cases being in dogs of the German Shepherd breed [6]. Another study used MRI to assess vertebrae T1 through T9 in 23 German shepherd breed dogs and 47 dogs of other breeds weighing more than $20 \mathrm{~kg}$. It was observed that although the two groups showed disc degeneration in the thoracic vertebrae, German Shepherds presented a higher number of degenerated discs located between $\mathrm{T} 2$ and $\mathrm{T} 5$ resulting in spinal cord compression. Thus, genetic, anatomical or conformational factors may be linked to the problem in German Shepherds [4].

Disc extrusions often result in more severe clinical signs than protrusions, and occur acutely or subacutely, which is different from the case described here, because maybe the presence of the intercapital 
ligament permitted gradual extrusion allowing the spinal cord to adapt to the compression [10]. The clinical condition described by the owner 3 months before consultation could indicate the onset of the disc material exiting the disc, thus reinforcing our theory, or be caused by another lighter compression, as that found at disc L2-L3.

Despite surgical access to this region being described as more complex $[6,12]$ due to the presence and proximity of the rib head to the vertebral body and the possibility of injuring the intercostal muscles and causing pneumothorax, the hemilaminectomy and rib head excision at $\mathrm{T} 9$ were performed without any complications. The decompression surgery in this case was deemed the most appropriate treatment considering the degree of the patient's injury [11].
Thus, although unusual, the cranial thoracic region should not be overlooked as a possible site of occurrence of IVDD, since the diagnosis, treatment and prognosis are similar to those in patients with extrusions in the most common sites.

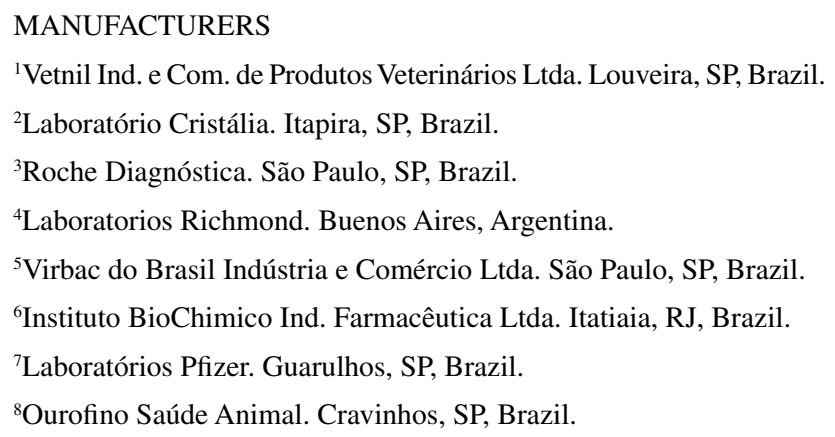

Declaration of interest. The authors report no conflicts of interest. The authors alone are responsible for the content and writing of the paper.

\section{REFERENCES}

1 Brisson B.A. 2010. Intervertebral disc disease in dogs. Veterinary Clinics of North America: Small Animal Practice. 40(5): 829-858.

2 Evans H.E. \& de Lahunta A. 2010. The skeletal and muscular systems. In: Guide to the Dissection of the Dog. 7th edn. St. Louis: Saunders Elsevier, pp.81-92.

3 Gaitero L. \& Añor S. 2009. Cranial thoracic disc protrusions in three German shepherd dogs. The Veterinary Journal. 182(2): 349-351.

4 Gaitero L., Nykamp S., Daniel R. \& Monteith G. 2013. Comparison between cranial thoracic intervertebral disc herniations in German Shepherd dogs and other large breed dogs. Veterinary Radiology \& Ultrasound. 54(2): 133-138.

5 Gilmore D.R. 1983. Cranial thoracic intervertebral disk extrusion in a dog. Journal of the American Veterinary Medical Association. 182(6): 620-621.

6 Hearon K., Berg J.M., Bonczynski J.J., Suarez C. \& Bergman P. 2014. Upper Thoracic Disc Disease (T1-T9) in Large-Breed Dogs. Journal of the American Animal Hospital Association. 50(2): 105-111.

7 Jäderlund K.H., Hansson K., Lindberg R. \& Narfström K. 2002. T3-T4 disc herniation in a German Shepherd Dog. Veterinary Record. 151(25): 769-770.

8 Liptak J.M., Watt P.R., Thomson M.J., Copeland S.E. \& Galloway A.M. 1999. Hansen type I disk disease at T1-2 in a dachshund. Australian Veterinary Journal. 77(3): 156-159.

9 Macias C., McKee W.M., May C. \& Innes J.F. 2002. Thoracolumbar disc disease in large dogs: a study of 99 cases. Journal of Small Animal Practice. 43(10): 439-446.

10 Olby N. \& Jeffery N. 2007. Pathogenesis of Diseases of the Central Nervous System. In: Textbook of Small Animal Surgery. v.1. 3rd edn. Philadelphia: Saunders, pp.1132-1147.

11 Sharp N.J.H. \& Wheeler S.J. 2005. Thoracolumbar disc disease. In: Small Animal Spinal Disorders Diagnosis and Surgery. 2nd edn. Philadelphia: Elsevier Mosby, pp.121-159.

12 Wilkens B.E., Selcer R., Adams W.H. \& Thomas W.B. 1996. T9-T10 Intervertebral disc herniation in three dogs. Veterinary and Comparative Orthopaedics and Traumatology. 9(4): 177-178. 